\title{
REVISÃO BIBLIOGRÁFICA NARRATIVA: \\ PLANEJAMENTO URBANO SAUDÁVEL
}

Literature review on healthy urban planning theme

http://dx.doi.org/10.21116/2017.1

\section{SPERANDIO, Ana Maria Girotti}

UNICAMP

Faculdade Jaguariúna

ROSA, Adriana Aparecida Carneiro

UNICAMP

Faculdade Jaguariúna

CARMO, Carolina Guida Cardoso do

UNICAMP

MONTREZOR, Danielle

UNICAMP

\section{CARVALHO, Fernanda Souza}

UNICAMP

\author{
ROCHA, Gisele \\ UNICAMP
}

TREVISAN, Simone Martins

UNICAMP

\section{BLOES, Rodrigo Brandini \\ UNICAMP}

\section{COCENZA, Vanessa}

UNICAMP

Resumo: A qualidade de vida e a promoção da saúde se mostram diretamente relacionadas ao planejamento urbano como fomentador de cidades saudáveis. Devido a relevância do tema para o atual cenário, a partir da necessidade de conhecimento da atual publicação científica relacionada a esta temática, foi realizada uma pesquisa de quantificação e sistematização de artigos científicos que abordam o tema em foco, para a partir desta pesquisa poder verificar ainda a quais áreas e instituições pertencem estes trabalhos publicados. Desta forma, este artigo visa apresentar um estudo realizado a partir da metodologia da revisão bibliográfica, ao longo de um período e quantidade de bases pré-determinados, a fim de delimitar um novo campo de estudo. Através da aplicação desta metodologia foi avaliada quantitativamente a atual produção bibliográfica na América Latina, dentro do recorte temporal entre os anos de 2005 e 2016. Ao longo do estabelecimento das etapas para a execução da pesquisa, que incluiu o inter-relacionamento das palavras-chave qualidade de vida, planejamento urbano e municípios saudáveis para determinar a 
abrangência da pesquisa, foi possível observar um crescimento progressivo de produção relacionadas à urbe saudável no período abordado pela pesquisa. Por outro lado, a pesquisa apontou uma restrição relacionada as áreas que abordam estas temáticas, havendo assim a necessidade de ampliação das mesmas, uma vez que a interdisciplinaridade é um dos pilares do tema aqui apresentado. Sendo assim, os resultados e reflexões provenientes deste processo de pesquisa, podem ser suporte para o desenvolvimento de outras pesquisas a medida em que as referências bibliográficas se mostram convergentes, sendo possível perceber a necessidade de pesquisas constantes.

Palavras-chave: Revisão Bibliográfica, Planejamento Urbano Saudável, Cidade Saudável.

Abstract: The quality of life and health promotion are directly related to urban planning as healthy cities promoter. In light of the theme relevance in the face of the current scenario, based on the need to know the current scientific publication related to this subject, a quantification and systematization research was carried out on scientific articles that approach the subject in focus, in addition to verify from which areas and institutions these published works are. In this way, this article reports a study based on the bibliographic review methodology, related to a predetermined period and scientific bases amount, in order to delimit a new field of study. From this methodology, it was evaluated the current bibliographic production in Latin America within the time frame between the years 2005 and 2016. Through the research stages establishment, which included the interrelationship of the keywords quality of life, urban planning and healthy municipalities to determine the study scope, it was possible to observe a progressive production growth related to the healthy city in the the period covered by study. On the other hand, the research pointed to a restriction related to the areas that deal with these issues, and thus the need for them to be expanded, since interdisciplinarity is one of the pillars of the theme presented here. Therefore, the results and reflections from this study can be a support to other researches development, insofar as the bibliographical references turned up convergent, being possible to perceive the need for constant research.

key-words: Bibliographic Review, Healthy Urban Planning, Healthy City.

\section{Introdução}

O planejamento urbano pode ser definido como um processo de organização e estruturação do espaço urbano, a fim de minimizar os conflitos das cidades e propor caminhos que propiciem uma melhora na qualidade de vida coletiva. $O$ Estatuto do da Cidade (BRASIL, 2001), documento constitucional norteador do planejamento urbano no Brasil, aponta como imprescindível para este processo ser bem sucedido a participação social, bem como o cumprimento da função social da cidade objetivando cidades mais saudáveis e equilibradas. 
É possível apontar, portanto, uma clara convergência entre o planejamento urbano e qualidade de vida. A convergência entre estas linhas de pesquisa é evidenciada na conceituação de município saudável, uma vez que o planejamento urbano pode promover a qualidade de vida estimulando a relação entre os cidadãos, seu entorno físico e social e o poder público, conforme apontado por Brandão (2010, p.37).

A conceituação de qualidade de vida, que está relacionada à satisfação obtida nas relações familiares, amorosas, sociais e ambientais, é intrinsicamente ligada a promoção da saúde, desde os séculos XVIII e XIX, no berço da medicina social (MINAYO, 2000). No âmbito internacional, a evolução do conceito de promoção da saúde, pautada pela busca da qualidade de vida concebida por Sigerist (1945), é visível como uma das tarefas dentre as ciências médicas.

Inicialmente, o conceito de promoção da saúde foi redimensionado pelo sanitarista canadense Lalonde, em 1974, considerando os conceitos atuais determinantes da saúde: I) estilo de vida; II) avanços na biologia humana; III) ambiente físico e social e IV) serviços de saúde (LALONDE, 1974, apud MINAYO, 2000). A partir desta reflexão e da relação de qualidade de vida e ambiente físico e social projetado, se faz necessária a discussão e constante atualização das referências mundiais relacionadas ao planejamento urbano saudável.

No Brasil, a difusão do movimento da promoção da saúde ocorre em um contexto marcado pela implementação de políticas públicas, programas sociais e projetos de reforma na organização e gestão das ações e serviços públicos de saúde, especialmente no âmbito municipal, lócus privilegiado de experimentação de alternativas ao modelo médico-assistencial hegemônico (MINAYO, 2000). No entanto, é preciso considerar que a promoção da saúde não está relacionada apenas ao tratamento de doenças e sim com a manutenção da qualidade de vida através da melhoria nas condições de habitação, lazer, alimentação, trabalho e renda entre outros determinantes sociais e urbanos. (BUSS, 2000)

A partir de 2006, com a implantação da Política Nacional de Promoção da Saúde (PNaPS), criou-se um compromisso legal da gestão federal em qualificar e ampliar as ações de promoção da saúde. Este compromisso foi reforçado com sua revisão no ano de 2014, evidenciando a importância da intersetorialidade, como objetivo e ferramenta de ação na promoção da qualidade de vida. 
A Política reforça a necessidade de articulação com outras políticas públicas para a promoção da saúde, com o imperativo da participação social e dos movimentos populares, assim como apontado pelo Estatuto da Cidade (BRASIL, 2001), em virtude da impossibilidade do setor sanitário responder sozinho ao enfrentamento dos determinantes e condicionantes da saúde. Assim, objetivos, princípios, valores, diretrizes, temas transversais, estratégias operacionais, responsabilidades e temas prioritários, reformulados e atualizados para esta política do Estado brasileiro, visam à equidade, à melhoria das condições e dos modos de viver e à afirmação do direito à vida e à saúde, dialogando com as reflexões dos movimentos no âmbito da promoção da saúde, fortalecendo a relação entre a comunidade e cidade. (BRASIL, 2014)

O cenário apresentado aponta o surgimento de uma nova corrente de pensamento, ações e necessidades. Diante disto são notáveis os estudos que exploram a inter-relação entre planejamento urbano e promoção da saúde, bem como suas reverberações na qualidade de vida coletiva e é formado o conceito de Planejamento Urbano Saudável.

Entende-se como Planejamento Urbano Saudável um conjunto de ações que visam tornar a cidade um local melhor para viver, considerando as premissas da Organização Mundial da Saúde, que avalia o planejamento urbano como essencial para o desenvolvimento da vida saudável (ROCHA et al, 2008). O Planejamento Urbano Saudável deve, para isso, considerar as características intrínsecas de uma cidade, as pessoas que nela vivem, bem como utilizar estratégias para formação do capital humano, participação social, governança, afetividade, dentre outras com o foco do bem viver e ser saudável. (SPERANDIO, 2012)

Esta intenção da política urbana é evidenciado com o discurso de Nardi (In: SPERANDIO, 2010), o qual afirma que a construção de Municípios Saudáveis só é possível quando a discussão da saúde contempla o território, promovendo uma ação intersetorial que garanta a qualidade de vida da população, utilizando o planejamento urbano como instrumento de proteção à vida, sendo assim, através de um Plano Diretor Participativo poderá ser concebida uma cidade que atenda às demandas sociais do território.

A fim de desenvolver pesquisas explorando a inter-relação entre planejamento urbano e a promoção da saúde, e atender a uma demanda crescente 
de conhecimento nesta área, o Laboratório de Investigações Urbanas (LABINUR), da Faculdade de Engenharia Civil, Arquitetura e Urbanismo (FEC) da Universidade Estadual de Campinas (UNICAMP), criou a linha de pesquisa em Planejamento Urbano e Cidade Saudável. A partir do objetivo de desencadear reflexões e estudos neste campo de conhecimento, foi apontada, ao longo das discussões em grupo, a necessidade de levantar a quantidade de pesquisas relacionadas à temática estudada.

Esse artigo pretende, portanto, apresentar os resultados de uma pesquisa realizada a partir da revisão bibliográfica narrativa dos últimos 10 anos de publicação científica relacionada a temática de planejamento urbano e promoção da saúde com enfoque na qualidade de vida.

\section{Metodologia}

O caráter da pesquisa foi quantitativo, com o objetivo de levantar numericamente a produção referente ao assunto e trazer possíveis reflexões a partir dos resultados obtidos.

De acordo com Moreira (2004), o primeiro passo para desenvolver uma revisão bibliográfica de qualidade é torná-la compreensível, sendo imprescindível conhecer as bases e bibliotecas disponíveis dentro do âmbito da pesquisa. É importante planejar a condução da pesquisa e seguir uma sequência esquematizada de indagações, como descrito a seguir: I) escolha do assunto e objetivos, analisando a utilidade do campo da pesquisa; II) levantamento bibliográfico, onde delimita-se as bases que farão parte da revisão; III) leitura inspecional, identificando quais textos serão analisados profundamente e a prioridade de leitura; IV) avaliação crítica, incluindo comparação e identificação de concordâncias e discordâncias entre os autores analisados. MOREIRA (2004)

Desta forma, a etapa inicial do projeto foi a determinação dos critérios chaves para a análise, apurando e refinando a abrangência da pesquisa à temática, os períodos, as bases de pesquisa e a localização espacial das produções acadêmicas, assim consideradas como teses, dissertações e artigos científicos. A delimitação temporal dos documentos foi traçada através da cronologia documental, considerando os materiais elaborados a partir do ano de 2005 até o primeiro semestre de 2016. 
A partir da necessidade de relacionar cidade saudável e qualidade de vida, a escolha das bases se limitou a seis bases de pesquisas disponíveis eletronicamente e de importância significativa para a comunidade científica Latino-americana. Tais bases foram acessadas durante os meses de Setembro e Outubro de 2015 e posteriormente em Outubro de 2016, determinados como o período de levantamento de dados da pesquisa. São elas:

I) Banco de Dados Bibliográficos da USP - Dedalus

II) Biblioteca Digital de Teses da UNICAMP

III) Portal de Periódicos da CAPES - CAPES Periódicos

IV) Scientific Electronic Library Online - Scielo

V) Google Acadêmico

VI) Sistema Regional de Información en Línea para Revistas Científicas de América Latina, el Caribe, España y Portugal - LATINDEX.

Conforme Dane, 1990 (apud CONFORTO et al, 2011) os critérios utilizados para referenciamento da pesquisa precisam ser preliminarmente determinados, como tópicos chaves a serem buscados, autores, fonte dos dados, de forma a especificar claramente a abrangência da revisão. Seguindo estas instruções, em uma segunda etapa foram adotadas quatro expressões para a busca a fim de contemplar de forma abrangente o maior espectro possível de trabalhos publicados. As expressões eleitas foram: "planejamento urbano saudável", "municípios saudáveis", "cidades saudáveis" e "qualidade de vida em planejamento urbano" a busca foi realizada em planejamento urbano e não a qualidade de vida dissociada do planejamento, para não ter áreas diferentes como educação física.

Após a sistematização dos resultados, organizados em tabelas, foram elaborados gráficos que apontam a cronologia e também as instituições de ensino de origem dos trabalhos que se enquadraram nos critérios já pré-estabelecidos.

Os trabalhos quantificados nesta pesquisa seguiram a mesma metodologia de busca, já apresentada, porém, dentro de cada texto, podem haver diferentes vertentes de pesquisas relacionadas à temática em foco. Alguns, por exemplo, não fazem referência a participação social para a promoção da cidade saudável, que 
conforme citado anteriormente, é uma das principais ferramentas a ser utilizadas para alcançar um planejamento urbano saudável.

\section{Resultados e Discussões}

A partir do levantamento dos dados seguindo a metodologia estabelecida, é possível observar que a quantidade de produções relacionadas ao tema do planejamento urbano saudável entre os anos de 2005 e o segundo semestre de 2016 estão vinculadas a grupos de pesquisa e pesquisadores participantes de laboratórios e instituições, em sua maioria, de âmbito público federal, conforme Gráfico 1.

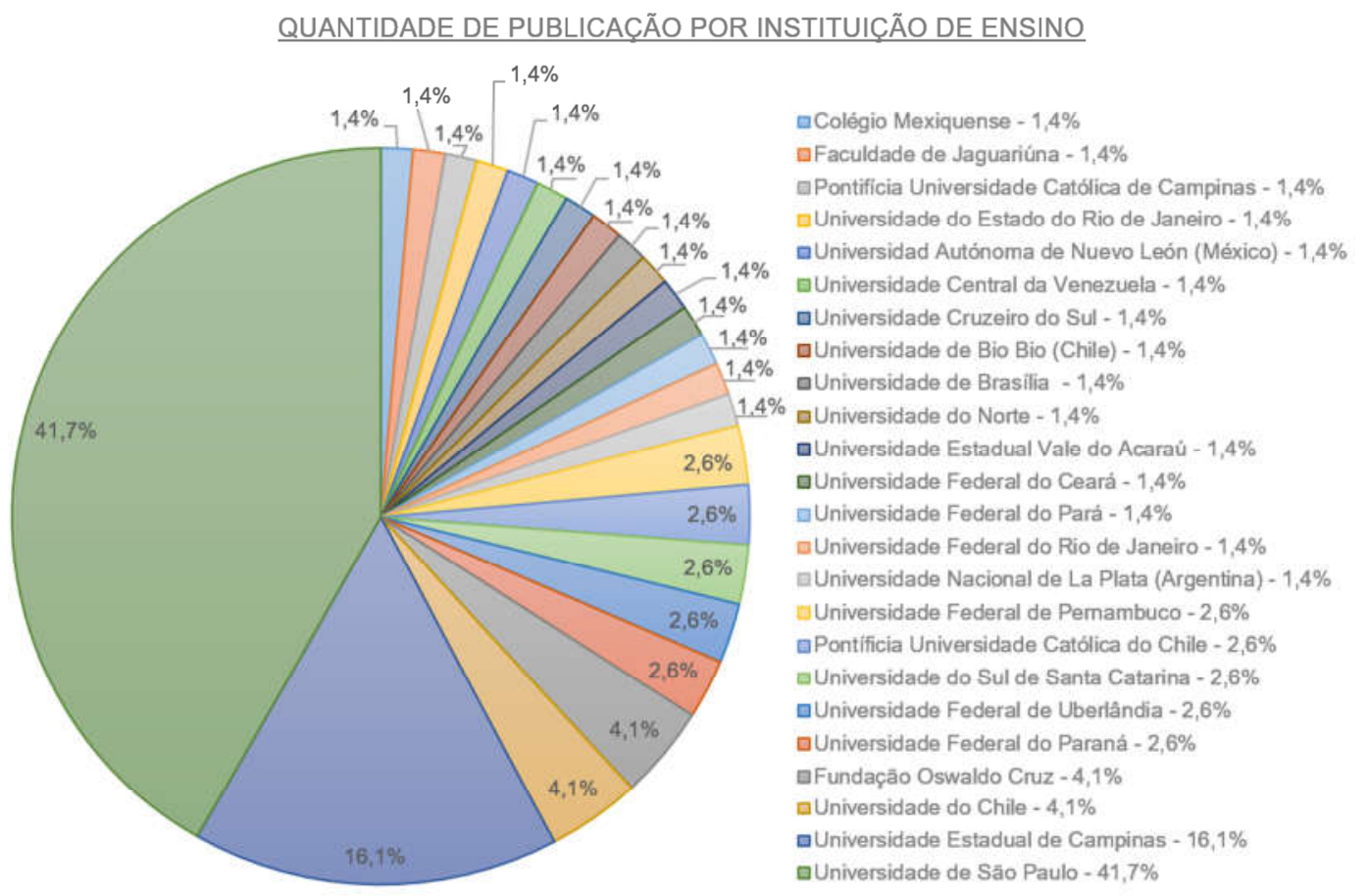

Gráfico 1. Publicações por Instituição de Ensino.

Fonte: Elaboração própria, 2016.

Através da localização das instituições, é possível espacializar geograficamente de maneira ilustrativa a produção na América do Sul, conforme Figura 1, onde se torna visível, a partir do método utilizado para buscar os artigos científicos, uma concentração de produções brasileiras, principalmente na região sudoeste do país, onde há um agrupamento de instituições com ampla trajetória em produção científica, como Universidade Estadual de Campinas (UNICAMP) e Universidade de São Paulo (USP). 


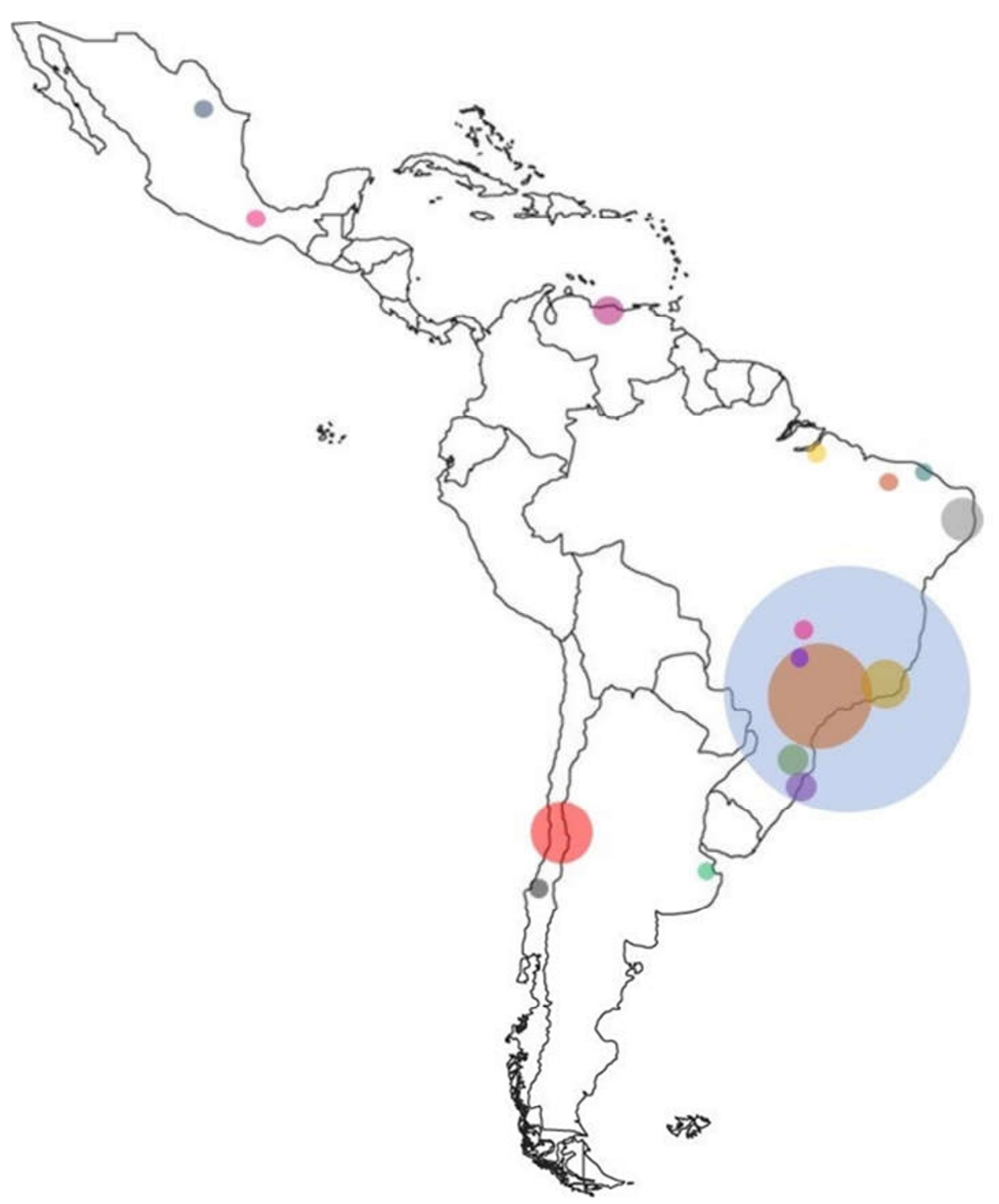

Figura 1. Mapeamento geográfico das produções acadêmicas. Fonte: Elaboração própria, 2016.

Nota-se também que, mesmo o planejamento urbano sendo pautado como interdisciplinar, norteado por documentos oficiais que enfatizam o envolvimento de diversos agentes e áreas do conhecimento, as pesquisas estão em sua maioria diretamente ligadas às áreas da saúde, especialmente dentro das Faculdades de Saúde Pública e centros de estudo voltados à saúde, conforma ilustrado no Gráfico 2. 


\section{PUBLICAČ̃̃ POR ÁREA}

\section{口Saúde $-45 \%$ \\ aPlanejamento Urbano - $40 \%$}

Demais áreas- $15 \%$

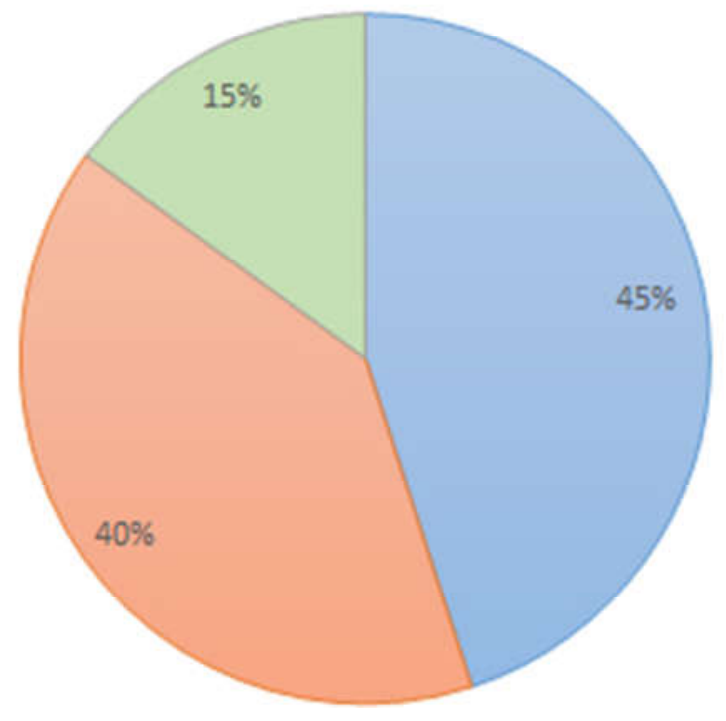

Gráfico 2. Publicações por áreas de atuação.

Fonte: Elaboração própria, 2016.

Para exemplificar um dos trabalhos relacionado à saúde: "Podemos Falar De Ambiente E Saúde Problematizando As Conexões Entre Saúde E Desenvolvimento?" (AKERMANN, 2008), trata a questão social urbana relacionada à saúde, porém não propõe o planejamento urbano como forma de elevar a saúde seja no âmbito físico ou emocional. Em outro, "A importância do Plano Diretor como ferramenta para construção de políticas públicas por um município potencialmente saudável" (SILVA, 2011) verifica a promoção da saúde através das políticas públicas como uma estratégia de transformação social saudável, a partir de um dos principais instrumentos do planejamento urbano, o Plano Diretor.

Também foi possível observar durante a pesquisa, conforme apresentado no Gráfico 3, que entre os anos de 2005 e 2016 houve um crescimento de publicações científicas relacionadas à temática. Entretanto, este crescimento não seguiu um padrão progressivo constante, havendo picos de produção no anos de 2008, 2011 e 2014, sendo este último o ano com maior quantidade de publicação dentro do período estudado. 


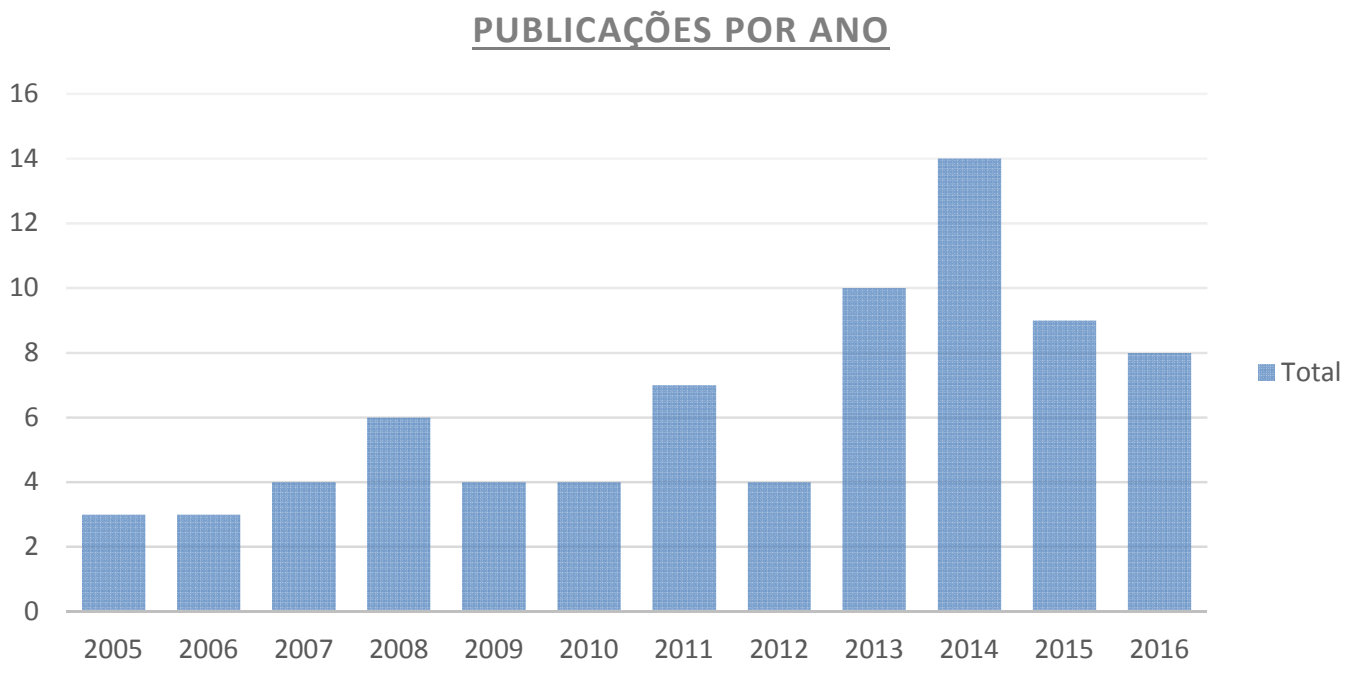

Gráfico 3. Quantificação anual de publicações

Fonte: Elaboração própria, 2016.

A aplicação da metodologia para obter os resultados esperados também foi avaliada como resultado do artigo. Observa-se pontos passíveis de mudanças para outras pesquisas do gênero em busca de outros resultados, como a ampliação das possibilidades de palavras chaves e melhorias nos usos dos filtros das próprias bases de pesquisa.

É importante ressaltar que todos os trabalhos acadêmicos considerados trazem à pauta a discussão do planejamento urbano saudável, apresentando em grande maioria, a relação do bem-estar e qualidade de vida dos usuários à manutenção e promoção da saúde, através de ferramentas do planejamento urbano e políticas de gestão. Desta maneira, as produções tendem a discutir as cidades saudáveis como um paradigma da promoção da saúde, apresentando e discutindo as condições necessárias para o desenvolvimento do saudável, através do compromisso de autoridades das gestões, estabelecendo metas, estratégias e objetivos relacionados às necessidades da população que vive nas cidades. 


\section{Considerações Finais}

Os conceitos de 'cidades saudáveis' e 'planejamento urbano saudável' foram formados recentemente em decorrência de uma demanda da área da saúde pública para solucionar problemas relacionados à saúde da população.

Este termo busca, de maneira geral, a reestruturação do espaço urbano a partir de um processo de participação social e de planejamento urbano, pois não se trata de saúde de forma setorial, deve-se agregar a cidade para melhor qualidade de vida das pessoas que nela vivem.

A partir dos resultados, pode-se apontar uma tendência de ampliação na quantidade de produção relacionado ao tema de planejamento urbano saudável, uma vez que foi observado um crescimento considerável das mesmas, direcionando o pesquisador ao entendimento da temática que se mostra cada vez mais presente, necessária e avaliada de grande importância na academia. Pode-se considerar assim, que o movimento das cidades saudáveis mostra-se, nestes artigos, como uma visão da promoção da saúde, incorporando estratégias de outros setores na sua formulação, reforçando o conceito de intersetorialidade.

Diante deste contexto, conforme apontado, as pesquisas científicas relacionadas ao tema ainda se concentram em algumas áreas da academia, como nas Faculdades de Saúde Pública. Desta maneira, a organização do material já produzido é válido a todo o processo de pesquisa, principalmente quando se deseja trabalhar com uma grande quantidade de dados. Além disso, os conceitos aqui abordados possuem um caráter de intersetorialidade e necessitam da ampliação da discussão em diversas áreas acadêmicas, como nas Faculdades de Arquitetura e Urbanismo, Educação, Economia e Geografia. 


\section{REFERÊNCIAS}

BRASIL. Estatuto da Cidade: Lei 10.257/2001 que estabelece diretrizes gerais da política urbana. Brasília, Câmara dos Deputados, 2001, 1a Edição.

BRASIL. PORTARIA No 2.446, DE 11 DE NOVEMBRO DE 2014. Redefine a Política Nacional de Promoção da Saúde (PNPS).

BUSS, P. M. Promoción de la salud y la salud pública: una contribución para el debate entre las escuelas de salud pública de América Latina y el Caribe. Rio de Janeiro, 2000. In: TEIXEIRA, Carmen Fontes; PAIM, Jairnilso Silva. Planejamento e programação de ações intersetoriais para a promoção da saúde e da qualidade de vida. RAP.Rio de Janeiro 34(6):63-80, Nov./Dez. 2000

Carta de Ottawa. Primeira Conferência Internacional sobre a Promoção da Saúde, Ottawa,1986. Disponível em http://bvsms.saude.gov.br/bvs/publicacoes/carta_ottawa.pdf. Consultada em 05/12/2015.

DANE, F. Research methods. Brooks/Cole Publishing Company: California, 1990. In: CONFORTO, E. C. ; AMARAL, D.C. ; SILVA, S.L. . Roteiro para Revisão Bibliográfica Sistemática: aplicação no desenvolvimento de produtos e gerenciamento de projetos. In: 8o. Congresso Brasileiro de Gestão de Desenvolvimento de Produto - CBGDP 2011, 2011, Porto Alegre-RS. 8o. Congresso Brasileiro de Gestão de Desenvolvimento de Produto - CBGDP 2011. Porto Alegre: Instituto de Gestão de Desenvolvimento de Produto, 2011

FIGUEIREDO, Nice. Da importância dos artigos de revisão da literatura. Revista Brasileira de Biblioteconomia e Documentação, São Paulo, v. 23, n. 1/4, p. 131-135, jan./dez. 1990.

HENRY E. Sigerist. Civilization and disease. Ithaca: Cornell University Press, 1945. Civilização e doença, trad. Marcos Fernandes da Silva Moreira. São Paulo: HucitecSobravime / Campinas: SindiMed, 2011. LALONDE M 1978. A New Perspective on the Health of Canadians: a Work Paper, 1974. Otawa. 76 pp. In: MINAYO, Maria Cecilia. Qualidade de Vida e Saúde: um debate necessário. Ciência \& Saúde Coletiva, 5(1): 7-18, 2000.

MOREIRA, Walter. Revisão de Literatura e Desenvolvimento Científico: conceitos e estratégias para confecção. Janus, Lorena, ano $1, n^{\circ} 1,2^{\circ}$ semestre de 2004

MINAYO, Maria Cecilia. Qualidade de Vida e Saúde: um debate necessário. Ciência \& Saúde Coletiva, 5(1): 7-18, 2000.

ROCHA, Daniel Pereira; PARNAIBA, Joseane Maria; SILVA, Maria Lucia; SPERANDIO, Ana Maria Girotti, FRANCISCO FILHO, Lauro Luiz. Programa de Aceleração do Crescimento (PAC) como gerador de qualidade de vida e saúde urbana: Relatos de experiências em Campinas-SP. Revista Intellectus, Ano VIII, $n^{\circ} 22,2012$. 
SPERANDIO, A.M.G. Organização Pan-Americana da Saúde. Livro: Políticas Integradas em Rede e a Construção de Espaços Saudáveis: Boas Práticas para a Iniciativa do Rostos, Vozes e Lugares. 2010.

\section{AUTORES:}

Professora Dra. Ana Maria Girotti Sperandio: Pesquisadora do Laboratório de Investigações Urbanas (LABINUR/FEC/UNICAMP) e Assessora Acadêmica e Coordenadora Geral do Núcleo de Estudos e Pesquisas da Faculdade Jaguariúna. amgspera@uol.com.br

Adriana Aparecida Carneiro Rosa: Arquiteta e Urbanista, Mestre em Engenharia Civil pela Universidade Estadual de Campinas (FEC-UNICAMP) e Professora no curso de Arquitetura e Urbanismo da Faculdade Jaguariúna.

dricarneiro@hotmail.com

Carolina do Carmo: Arquiteta e Urbanista, aluna de Mestrado do Programa Arquitetura, Tecnologia e Cidade (FEC-UNICAMP).

carolinagcdocarmo@gmail.com

Danielle Montrezor: Arquiteta e Urbanista, aluna de Mestrado do Programa Arquitetura, Tecnologia e Cidade (FEC-UNICAMP).

dmontrezor.arq@gmail.com

Fernanda Carvalho: Engenheira Civil, aluna de Mestrado na Faculdade de Tecnologia da UNICAMP.

fer_unicamp@yahoo.com

Gisele Rocha: Arquiteta e Urbanista

arq.giselerocha@gmail.com

Rodrigo Brandini Bloes: Engenheiro Civil

rodrigo.bbloes@gmail.com

Simone Martins Trevisan: Arquitetura e Urbanista, Especialista em Gestão Ambiental e Desenvolvimento Sustentável.

sicatrevisan@gmail.com

Vanessa Cocenza: Arquiteta e Urbanista, aluna de Mestrado do Programa Arquitetura, Tecnologia e Cidade (FEC-UNICAMP).

vanessa.cocenza@gmail.com 